\title{
Elucidating Ion Transport in Lithium-Ion Conductors by Combining Vibrational Spectroscopy in STEM and Neutron Scattering
}

\author{
Miaofang Chi ${ }^{1}$, Xiaoming Liu ${ }^{1}$, Jordan Hachtel $^{1}$, Niina H. Jalarvo ${ }^{2}$, Yongqiang Cheng ${ }^{2}$, and Jeff \\ Sakamoto $^{3}$ \\ 1. Center for Nanophase Materials Sciences, Oak Ridge National Laboratory, Oak Ridge, TN, USA. \\ 2. Neutron Scattering Division, Oak Ridge National Laboratory, Oak Ridge, TN, USA. \\ 3. Mechanical Engineering, University of Michigan, Ann Arbor, MI, USA.
}

Lithium-ion batteries are currently one of the leading electrochemical energy storage techniques for many transportation and stationary applications. However, the use of liquid electrolytes negatively effects their capacity, lifetime, and safety. Solid-state electrolytes are being investigated as one of the best solutions to overcome these challenges and are considered as a critical component in futuregeneration lithium battery systems, which includes their use as the primary electrolytes in all-solid-state batteries, and the interfacial separation layer for Li-air or aqueous batteries [1]. Unfortunately, the ionic conductivity of many solid electrolytes is much lower than that of their liquid counterparts, largely due to slow transport kinetics across the interfaces associated with solid electrolytes, e.g., grain boundaries and interfaces formed with electrodes [2]. Furthermore, the ion conduction behavior in solid electrolytes in the presence of other mobile ions during operation, such as protons in the Li-air or aqueous batteries, is not understood.

Ion transport in solids is primary determined by the concentration and distribution of mobile ions, the connection of ion hopping channels, and the activation energy required for ions to hop. Lattice, chemistry, and ion diffusion must be correlatively studied. While lattice and chemistry can be elucidated by high-resolution imaging and electron energy loss spectroscopy (EELS) in a scanning transmission electron microscope (STEM), the diffusion behavior of ions cannot be determined in a straightforward manner [3]. On the other hand, neutron scattering (NS) is capable of providing information about ion diffusion, but is limited to averaged "bulk" diffusion behavior, and thus, probing the ion behavior of local features such as interfaces is challenging [4]. NS is based on the interaction between the neutrons and the atomic nuclei in the studied material, and in particular, inelastic neutron scattering (INS) can provide information regarding bonding potentials that affect the activation energy of mobile ions. Quasielastic INS, on the other hand, can be used to extract the mean square displacements of mobile ions, and therefore, the activation energy for ion hopping. Due to the recent development of high energy resolution monochromation, vibrational spectroscopy in STEM is now possible, creating new opportunities to study ion diffusion at features that are spatially confined, such as boundaries and interfaces $[5,6]$. By combining the complementary characterization methods of STEM and NS, ion diffusion behavior at local features and within the bulk material can be probed and correlated.

In this work, a protonated ceramic solid electrolyte, $\left(\mathrm{Li}_{6.25} \mathrm{Al}_{0.25}\right) \mathrm{La}_{3} \mathrm{Zr}_{2} \mathrm{O}_{12}$ (LLZO) was used to demonstrate the power of combining imaging and vibrational spectroscopy in STEM, and inelastic and elastic scattering in NS, to elucidate dual ion diffusion, i.e., $\mathrm{Li}^{+}$and $\mathrm{H}^{+}$ions, in the LLZO lattice and at its grain boundaries. The combination of STEM imaging (Fig. 1a) and NS revealed the concentration of $\mathrm{H}^{+}$ions in the lattice as well as the specific lattice sites they occupy (Fig. 1b). A slight structural distortion was observed as a result of ion replacement of $\mathrm{Li}^{+}$with $\mathrm{H}^{+}$. Quasi-elastic scattering revealed two distinct diffusion behaviors in protonated LLZO, allowing for differentiation of the contributions from $\mathrm{H}^{+}$and $\mathrm{Li}^{+}$. Vibration spectroscopy in STEM clearly revealed the vibration mode of $\mathrm{H}^{+}$at 
$\sim 150 \mathrm{meV}$. The vibration peak of $\mathrm{H}^{+}$was found to change with concentration and the site occupancy of $\mathrm{H}^{+}$ions in LLZO. Furthermore, it was observed that the presence of $\mathrm{H}^{+}$in LLZO induced a slower lithium diffusion, in contrast to that observed in anti-perovskite lithium ion conductors, where the presence of $\mathrm{H}^{+}$assisted $\mathrm{Li}^{+}$ion conduction. The vibration spectra of LLZO grain boundaries will be discussed and compared to that from the LLZO bulk.

\section{References:}

[1] C Ma and M Chi, Front. Energy Res. 4 (2016), p. 23.

[2] C Ma, K Chen, CD Liang, et al., Energy \& Environmental Science 7 (2014), p. 1638.

[3] C Ma, Y Cheng, K Chen, et al., Adv. Energy Mater. 6 (2016) p. 1600053.

[4] YQ Cheng, J Balachandran, ZH Bi, et al., J. Mater. Chem. A 5 (2017), 15507.

[5] OL Krivanek, TC Lovejoy, N Dellby, et al., Nature 514 (2014), p. 209.

[6] P Rez, T Aoki, K March, et al., Nature Comm. 7 (2016).

[4] Research conducted as part of a user project at ORNL's Center for Nanophase Materials Sciences, and neutron scattering experiments were performed at the VULCAN, VISION, and BASIS beamlines at ORNL's Spallation Neutron Source, both of which are U.S. DOE Office of Science User Facilities.

\section{(a)}

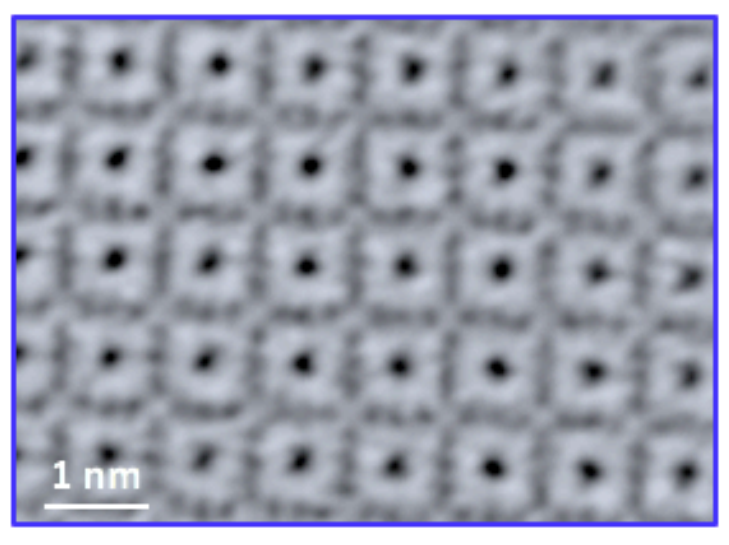

(b)

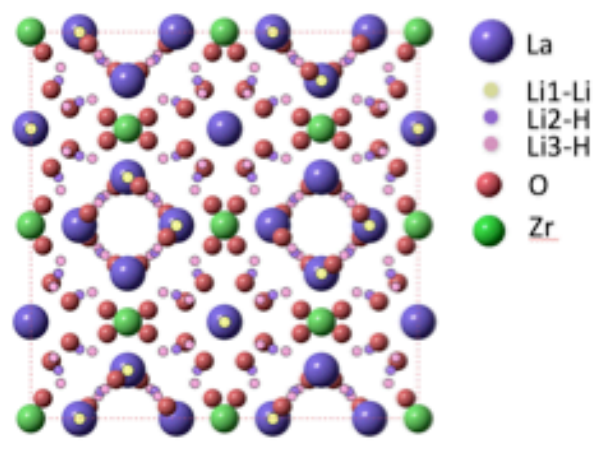

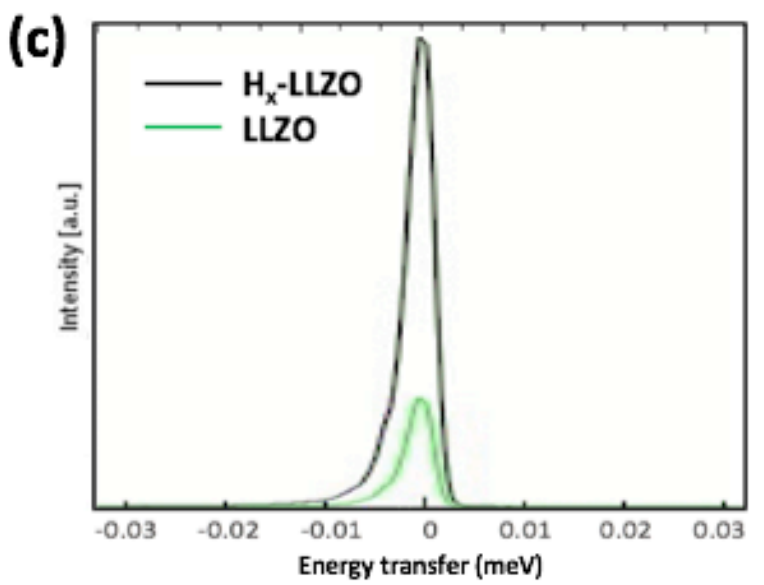

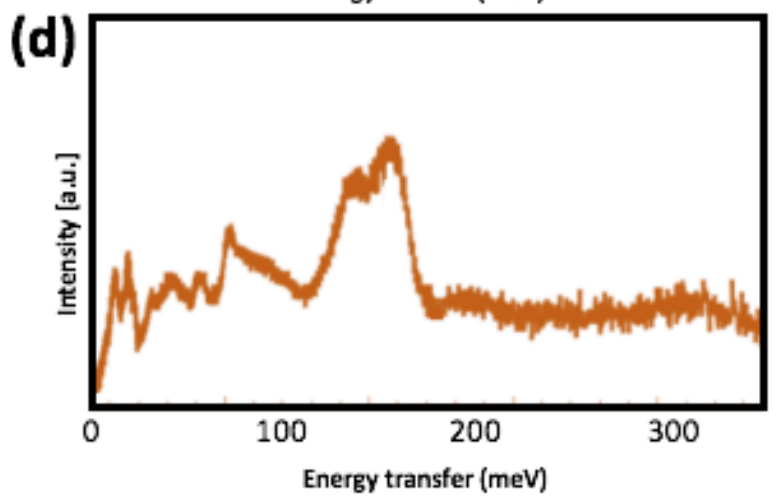

Figure 1. (a) STEM annular bright field (ABF) image of H-LLZO; (b) H occupancy at Li sites in LLZO determined by combining results from high angle annular dark field (HAADF)-STEM imaging, EELS, and neutron diffraction; (c) quasi-elastic scattering spectra of LLZO and H-LLZO summed over Q range 0.2-1.0 $\AA^{-1}$ free of Bragg peaks, indicating different scattering probabilities of $\mathrm{H}+$ and $\mathrm{Li}+$; (d) vibrational spectroscopy of LLZO acquired by using inelastic neutron scattering. 